\title{
Industry quantum computing applications
}

Quantum Technology and Application Consortium - QUTAC $^{1 *}$, Andreas Bayerstadler ${ }^{8}$, Guillaume Becquin ${ }^{8}$, Julia Binder ${ }^{6}$, Thierry Botter ${ }^{12}$, Hans Ehm ${ }^{6}$, Thomas Ehmer ${ }^{7}$, Marvin Erdmann³, Norbert Gaus ${ }^{10}$, Philipp Harbach7, Maximilian Hess ${ }^{6}$, Johannes Klepsch ${ }^{3}$ (1) Martin Leib ${ }^{11}$, Sebastian Luber ${ }^{6}$, Andre Luckow ${ }^{3}$, Maximilian Mansky ${ }^{7}$, Wolfgang Mauerer ${ }^{10}$, Florian Neukart ${ }^{11}$, Christoph Niedermeier ${ }^{10}$, Lilly Palackal ${ }^{6}$, Ruben Pfeiffer ${ }^{6}$, Carsten Polenz ${ }^{9}$, Johanna Sepulveda ${ }^{12}$, Tammo Sievers ${ }^{6}$, Brian Standen², Michael Streif ${ }^{4}$, Thomas Strohm ${ }^{5}$, Clemens Utschig-Utschig ${ }^{4}$, Daniel Volz ${ }^{2}$, Horst Weiss ${ }^{2}$ and Fabian Winter ${ }^{8}$

"Correspondence: info@qutac.de ${ }^{1}$ QUTAC, Germany Full list of author information is available at the end of the article

\section{Springer}

\begin{abstract}
Quantum computing promises to overcome computational limitations with better and faster solutions for optimization, simulation, and machine learning problems. Europe and Germany are in the process of successfully establishing research and funding programs with the objective to advance the technology's ecosystem and industrialization, thereby ensuring digital sovereignty, security, and competitiveness. Such an ecosystem comprises hardware/software solution providers, system integrators, and users from research institutions, start-ups, and industry. The vision of the Quantum Technology and Application Consortium (QUTAC) is to establish and advance the quantum computing ecosystem, supporting the ambitious goals of the German government and various research programs. QUTAC is comprised of ten members representing different industries, in particular automotive manufacturing, chemical and pharmaceutical production, insurance, and technology. In this paper, we survey the current state of quantum computing in these sectors as well as the aerospace industry and identify the contributions of QUTAC to the ecosystem. We propose an application-centric approach for the industrialization of the technology based on proven business impact. This paper identifies 24 different use cases. By formalizing high-value use cases into well-described reference problems and benchmarks, we will guide technological progress and eventually commercialization. Our results will be beneficial to all ecosystem participants, including suppliers, system integrators, software developers, users, policymakers, funding program managers, and investors.
\end{abstract}

Keywords: Quantum computing; QUTAC; Industry application; Reference problems; Benchmarks

\section{Introduction}

With quantum computers surpassing leading supercomputers in specific computational challenges [1, 2], and the availability of noisy intermediate-scale quantum (NISQ)-era quantum computing systems [3-6] outside of laboratory environments, we have entered

(c) The Author(s) 2021. This article is licensed under a Creative Commons Attribution 4.0 International License, which permits use, sharing, adaptation, distribution and reproduction in any medium or format, as long as you give appropriate credit to the original author(s) and the source, provide a link to the Creative Commons licence, and indicate if changes were made. The images or other third party material in this article are included in the article's Creative Commons licence, unless indicated otherwise in a credit line to the material. If material is not included in the article's Creative Commons licence and your intended use is not permitted by statutory regulation or exceeds the permitted use, you will need to obtain permission directly from the copyright holder. To view a copy of this licence, visit http://creativecommons.org/licenses/by/4.0/. 
the industrialization stage of quantum computing $(\mathrm{QC})$. Globally, national research programs and private investors are heavily funding quantum technologies (e.g., UK [7], US [8-10], China [11]). Investments are motivated by the need to ensure digital sovereignty, national security, and sustain the industry's competitiveness.

Quantum ecosystems and markets are still in their infancy. As technology matures, the market will grow. BCG estimates that the market size will surpass $\$ 450$ billion annually in the next decade [12]. A crucial driver will be the real-world use of the technology within business applications. Quantum Computing promises to solve high-value, classically intractable computational problems in the domains of optimization, machine learning, and simulation across all industry sectors [13].

Europe needs a vibrant ecosystem to foster quantum computing development and compete on a global scale. With its internationally renowned research institutions engaged in foundational research [14] and strong industrial users [13], Germany is in an excellent position. However, industrialization in Europe has traditionally been hampered by the European paradox [15], referring to Europe's member states hosting world-leading scientific and technological research activities, but unable to convert these into global industrial and commercial leadership.

The European Union and nations are establishing various programs to foster attractive ecosystems and markets for quantum technologies [16-18]. While these programs focus in particular on research and hardware technology industrialization (e.g., superconducting, ion-trap, photonic and solid-state qubits), they also emphasize the importance of holistic ecosystems. These ecosystems are to align the entire value-chain, including hardware and software solution providers, investors, and especially industry [16], which is essential for progressing high-value use cases that can advance commercialization.

So far, industries have refrained from commitment due to high risks and delayed return on investment. The Quantum Application and Technology Consortium (QUTAC) addresses this issue by joining the efforts of ten industrial companies to advance the German quantum computing ecosystem. The primary effort is the contribution of potential applications and a business-centric perspective to emerging initiatives from both Germany and the European Union.

This paper is structured as follows: In Sect. 2, we provide an overview of QUTAC. We continue with discussing a portfolio of use cases from the German industry in Sect. 3, a core asset that will guide QUTAC's further activities in the ecosystem. We discuss challenges for further industrialization in Sect. 4. In Sect. 5, we conclude with a discussion a call for action.

\section{QUTAC: quantum application and technology consortium}

QUTAC aims to raise quantum computing to the level of large-scale industrial applications while preparing our members for a new digital future. We bring together the expertise of Germany's industry to efficiently advance quantum computing towards real-world applications, ensuring Germany's and Europe's digital sovereignty, national security, and competitiveness in a global economy.

QUTAC will move the emerging quantum computing ecosystem forward, supporting the ambitious goals of the German government. It comprises ten companies from four sectors (see Table 1) with the mission to contribute an industry perspective and focus on the development of the German and European quantum ecosystems. QUTAC focuses primarily on the applications of quantum computing. Our members share the need to act due 
Table 1 Industry Sectors and QUTAC members

\begin{tabular}{ll}
\hline Industry Sector & Companies \\
\hline Automotive Manufacturing & BMW, Bosch, Volkswagen \\
Chemical \& Pharmaceutical & BASF, Boehringer Ingelheim, Merck \\
Insurance & Munich Re \\
Technology & Infineon, SAP, Siemens \\
\hline
\end{tabular}

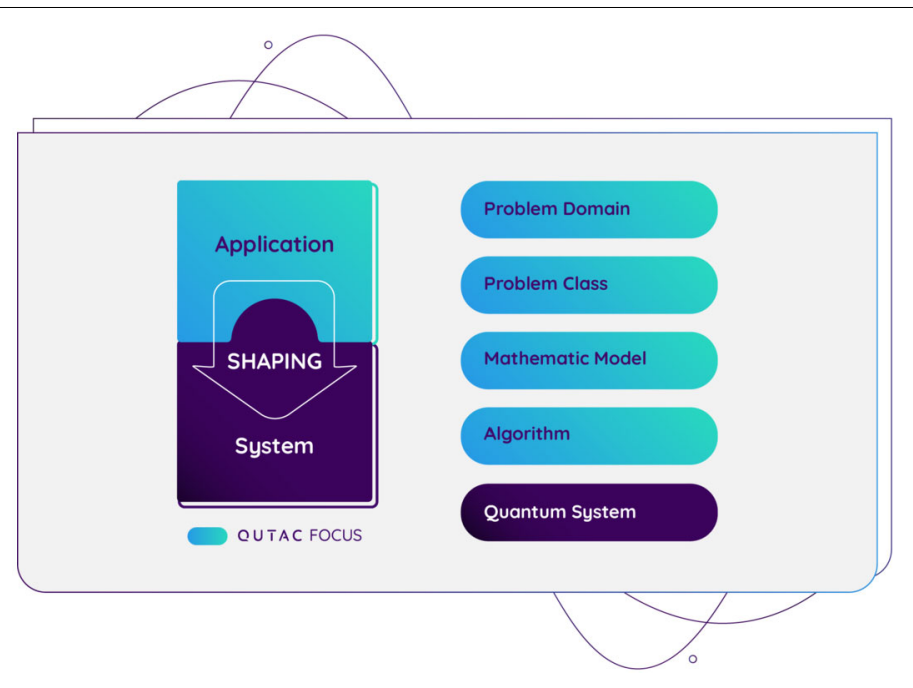

Figure 1 QUTAC Focus: QUTAC aims to shape the quantum computing ecosystem from the application perspective, balancing business impact and technological advances

to the potentially disruptive impact of quantum computing on all aspects of our business and value-chains. Collaboration across company borders promises to de-risk fundamental, pre-competitive research. Additionally, quantum computing might allow some members to explore further opportunities in the quantum value-chain, e.g., as a component or software provider.

Figure 1 illustrates the focus of QUTAC. The value-chain of QUTAC members comprises complex optimization, machine learning, and simulation challenges that are likely to benefit from advances in quantum computing, providing significant business impact. A wide variety of problems with impact across diverse industries will provide guidance to software and hardware development.

QUTAC will participate and contribute to the emerging European quantum ecosystem by collaborating closely with: (1) hardware solution providers, (2) component manufacturers, (3) software solution providers, (4) research institutions (public, private), (5) investors, and (6) end-users. Table 2 summarizes how QUTAC will provide value to all stakeholders.

QUTAC's guiding principles are:

- Promote the establishment of an economically thriving, independent quantum computing ecosystem in Germany and Europe.

- Raise awareness of the potential impact and competitive advantage that quantum technologies can provide across industries, motivating early investment and engagement.

- Understand, develop, and test cross-industry applications to identify commercially interesting solutions that can drive the quantum ecosystem forward. 
Table 2 QUTAC Stakeholder Assessment: QUTAC will make important contributions by providing an industry and application perspective to ecosystem stakeholders

\begin{tabular}{|c|c|}
\hline Ecosystem Stakeholder & QUTAC Contribution \\
\hline $\begin{array}{l}\text { Hardware solution provider/ } \\
\text { component supplier }\end{array}$ & $\begin{array}{l}\text { - Guidance on high-value use cases, reference problems and their business } \\
\text { impact } \\
\text { - Reference problems and benchmarks for assessing competitiveness of } \\
\text { approach } \\
\text { - Direct or indirect customer for future products }\end{array}$ \\
\hline Software solution provider & $\begin{array}{l}\text { - Assess the suitability of abstractions, frameworks, and services for industry } \\
\text { problems } \\
\text { - Guidance on end-to-end application workflows including both quantum und } \\
\text { classical steps } \\
\text { - Reference problems and benchmarks }\end{array}$ \\
\hline $\begin{array}{l}\text { Research institution and } \\
\text { program (e.g., Hubs, DLR, } \\
\text { Fraunhofer) }\end{array}$ & $\begin{array}{l}\text { - Guide application-centric research with reference problems and benchmarks } \\
\text { - Collaborative research and industrialization } \\
\text { - Joint ventures and spin-off opportunities }\end{array}$ \\
\hline Investor & $\begin{array}{l}\text { - Assess the viability of different approaches based on well-defined industry } \\
\text { problems }\end{array}$ \\
\hline Policy maker & $\begin{array}{l}\text { - Industry perspective for current and future research programs } \\
\text { - Assess the viability of different approaches based on well-defined industry } \\
\text { problems } \\
\text { - Reduce investment risks by early assessment of transfer opportunities } \\
\text { - Develop a multi-perspective research landscape across all stakeholders } \\
\text { - Explore new policies that increase ecosystem collaboration and } \\
\text { time-to-market }\end{array}$ \\
\hline QUTAC member & $\begin{array}{l}\text { - De-risk through pre-competitive collaborative research } \\
\text { - First-mover and competitive advantage for early access to technology } \\
\text { - Explore potential business opportunities in the quantum ecosystem }\end{array}$ \\
\hline
\end{tabular}

- Contribute to the success of the government's ambitious quantum program by providing a perspective from the industry and application angle.

QUTAC is organized through working groups that aim to identify commercially attractive solutions for high-impact business problems. QUTAC started with three operational working groups that are structured by the main challenges: "material science", "engineering", and "production \& logistics". Members share the need to understand, develop, and evaluate cross-industrial applications on emerging quantum hardware. Such crossindustrial problems include optimizations, machine learning and simulation challenges. QUTAC members present 24 use cases and common challenges in this paper.

\section{Industry applications}

While quantum computing will have a significant impact on various industries [12, 19], many questions and challenges remain: Which specific problems can be solved? Which of the NISQ quantum devices provide a quantum advantage? How can this advantage be translated into business impact? Here, we present an analysis of high-impact industry quantum applications. The analysis is based on several workshops, a structured survey (answered by 17 experts from 11 companies across 5 industry sectors), and interviews (9 bilateral interviews). The contributing companies have each shared up to three quantum computing use cases.

We identified the following layers: problem domain, problem class, model, algorithms, and quantum systems. To ensure consistency in cross-industry application discussions, we suggest the terminology defined in the following definition box. 


\section{Definitions}

- Problem Domains: Problem areas of applied mathematics and computer science characterized by similar solution methods aiming to solve computational problems (e.g., Optimization, Simulation, Machine Learning, Cryptography).

- Problem Class: A problem class is a set of use cases that share a similar mathematical formulation and the computational complexity class. It can be characterized by the common mathematical and business problem formulation (e.g., Software testing posed as 3-satisfiability problem).

- Model: A model is defined as a mathematical formulation describing a system capturing all practically relevant properties. Models are a simplified representation of reality supporting the understanding of its component's interactions and impact on resulting properties as well as predictions on future behavior (e.g., the train and driver recovery problem in a set partitioning problem formulation).

- Algorithm: A quantum algorithm is a finite sequence of quantum computer-implementable instructions to perform computations. They are typically used to find a solution or an approximation of a class of mathematically defined problems.

- Quantum System: A quantum computing system (short: quantum system) is a system for computation that makes direct use of quantum-mechanical phenomena (e.g., superposition, entanglement) to perform operations on data.

\subsection{Application overview}

General application challenges across the industrial sectors share the common problem domains, in particular optimization, machine learning, and simulation. In the following, we highlight selected challenges from our value-chains. We focus on four areas of value creation: (1) material science, (2) engineering \& design, (3) production \& logistic, and (4) post-quantum security. Table 3 summarizes the collected use cases.

\subsubsection{Material science}

Simulating and predicting the behavior of complex, quantum-mechanical systems is critical for new design, such as new types of batteries or pharmaceutical drugs. McKinsey \& Company predicts that quantum chemistry will be an early disruptive application of quantum computing [19]. Modeling polymers, solids, molecules at high precision without experimentally synthesizing materials in the lab enables identification of effective molecular structures that satisfy desirable properties such as high energy density or stiffness [20]. Classic examples include drug discovery or the Haber-Bosch process: Industrial production of chemicals such as the catalytic nitrogen fixation of ammonia in the Haber-Bosch process uses up $1 \%$ of the world's energy production and is responsible for $1.4 \%$ of the carbon-dioxide emission [21]. On a large scale, even relatively small improvements would cause a relevant absolute impact.

The global relevance of quantum computing in Material Science is also reflected in our working group. There are various QUTAC material science examples, including prediction of chemical reactivity in the chemical industry (BASF), molecular dynamics for drug discovery (Boehringer Ingelheim, Merck), and battery research (VW). 
Table 3 Initial Use Case Portfolio: A wide variety of optimization, simulation, and machine learning problems exist in the value-chains across the German industry. While impact in the next 5 years is low, several high-impact use cases have been identified

\begin{tabular}{|c|c|c|c|c|}
\hline Challenge & Problem Domain & Company & Use Case & Impact \\
\hline \multirow[t]{3}{*}{$\begin{array}{l}\text { Engineering } \\
\text { \& Design }\end{array}$} & Machine Learning & AIRBUS & $\begin{array}{l}\text { QC for Surrogate Modeling of Partial } \\
\text { Differential Equations }\end{array}$ & High \\
\hline & Optimization & $\begin{array}{l}\text { AIRBUS } \\
\text { Bosch }\end{array}$ & $\begin{array}{l}\text { Wingbox Design Optimization } \\
\text { Software Testing and Correctness Proving }\end{array}$ & $\begin{array}{l}\text { High } \\
\text { Medium }\end{array}$ \\
\hline & Simulation & Bosch & $\begin{array}{l}\text { Design Optimizations for Electric Drives } \\
\text { Using Numerical Simulation and Finite } \\
\text { Element Methods } \\
\text { Identification and control of Actionable } \\
\text { Parameters for Disease Spread Control }\end{array}$ & Medium \\
\hline \multirow[t]{6}{*}{$\begin{array}{l}\text { Material } \\
\text { Science }\end{array}$} & Optimization & $\begin{array}{l}\text { Boehringer } \\
\text { Ingelheim }\end{array}$ & $\begin{array}{l}\text { Optimized Imaging - Quantum-Inspired } \\
\text { Imaging Techniques }\end{array}$ & Medium \\
\hline & Simulation & BASF & $\begin{array}{l}\text { Quantum Chemistry - Prediction of } \\
\text { Chemical Reactivity in Molecular Quantum } \\
\text { Chemistry }\end{array}$ & High \\
\hline & & $\begin{array}{l}\text { Boehringer } \\
\text { Ingelheim }\end{array}$ & $\begin{array}{l}\text { Molecular Dynamics - Simulation of the } \\
\text { Dynamics of Molecules }\end{array}$ & High \\
\hline & & Merck & $\begin{array}{l}\text { Development of Materials and Drugs Using } \\
\text { Quantum Simulations }\end{array}$ & Medium \\
\hline & & Munich Re & $\begin{array}{l}\text { Battery Cover - Performance Guarantees for } \\
\text { eVehicle Batteries }\end{array}$ & Medium \\
\hline & & VW & Chemistry Calculation for Battery Research & High \\
\hline \multirow[t]{12}{*}{$\begin{array}{c}\text { Production \& } \\
\text { Logistics }\end{array}$} & Machine Learning & Siemens & $\begin{array}{l}\text { QaRL - Quantum-assisted Reinforcement } \\
\text { Learning - Applicable to many Industrial } \\
\text { Use Cases }\end{array}$ & Medium \\
\hline & Optimization & BASF & $\begin{array}{l}\text { Fleet Management - On-site Truck and } \\
\text { Machine Deployment and Routing }\end{array}$ & Medium \\
\hline & & BMW & $\begin{array}{l}\text { Robot Production Planning - Robot path } \\
\text { Optimization for Production Robots (e.g., } \\
\text { PVC sealing robot) }\end{array}$ & Medium \\
\hline & & BMW & $\begin{array}{l}\text { Vehicle Feature Testing - Optimizing Test } \\
\text { Vehicle Option Configuration }\end{array}$ & Medium \\
\hline & & BMW & $\begin{array}{l}\text { Shift Scheduling - Optimizing Labour Shift } \\
\text { Assignments }\end{array}$ & Medium \\
\hline & & Infineon & $\begin{array}{l}\text { Demand Capacity Match in Supply Chain - } \\
\text { Decide on a Production Plan given } \\
\text { Predicted Customer Demand }\end{array}$ & Medium \\
\hline & & Infineon & $\begin{array}{l}\text { Using Infineon Sensors and Actuators to } \\
\text { Optimize Supply Chain Processes on the } \\
\text { Customer Side }\end{array}$ & Medium \\
\hline & & Munich Re & $\begin{array}{l}\text { Transportation Cover - Insurance of } \\
\text { Time-Critical Freight }\end{array}$ & Medium \\
\hline & & SAP & Logistics - Truck Loading & Medium \\
\hline & & SAP & $\begin{array}{l}\text { Supply Chain Planning - Improved and } \\
\text { Accelerated Sizing of Orders (Lot Sizing) }\end{array}$ & High \\
\hline & & Siemens & $\begin{array}{l}\text { QoMP - Quantum-optimized Matrix } \\
\text { Production - Realtime Shop Floor } \\
\text { Optimization }\end{array}$ & Medium \\
\hline & & VW & $\begin{array}{l}\text { Vehicle Routing Problem - Optimize Vehicle } \\
\text { Utilization in a Transport Network }\end{array}$ & High \\
\hline $\begin{array}{l}\text { Post-Quantum } \\
\text { Security }\end{array}$ & Cryptography & Munich Re & $\begin{array}{l}\text { loT Cyber Cover - Insurance of Post } \\
\text { Quantum Cryptography }\end{array}$ & Medium \\
\hline
\end{tabular}

\subsubsection{Engineering $\mathcal{E}$ design}

Engineering simulations are heavily used across the contributors of this paper, particularly in the manufacturing sector. Such simulations are crucial to decrease efforts for design 
and testing by reducing the necessity of physical prototypes and laboratories, e.g., wind tunnels in the automotive and aerospace domain. Current in-silico models are limited by the complexity and quality of supported models and the necessary compute time.

Numerical simulations, particularly finite-element-method (FEM)-based, are crucial to simulate complex processes such as aerodynamics, operating strength, structural dynamics, crash \& safety, and production concerns [22]. For example, Bosch is investigating QCbased simulation approaches for electric drives.

Furthermore, AIRBUS is exploring the usage of quantum or hybrid quantum-classical approaches for computational fluid dynamics to reduce the computational resources required to analyze the behavior of airflow around the aircraft. Finally, research approaches, such as the usage of surrogate machine-learning-based models for numerical simulations (AIRBUS), are being investigated [23]. Another important problem domain is design optimization. An example is the design of aircraft wingboxes (AIRBUS) [24].

Solutions require various factors to be assessed simultaneously to ensure that structural integrity is maintained. As a result, current processes to address the problem are inefficient and require significant computational resources with long design times. This problem is exacerbated by more advanced and computationally intensive generative design methods that are increasingly explored across industries.

\subsubsection{Production E logistics}

Optimization and simulation problems are omnipresent in the production \& logistics domain across all industries, i.e. manufacturing, chemical \& pharmaceutical production, insurance and technology. Examples of common problems are routing, supply chain, production planning, and insurance risk assessment. Real-world problems often involve a large number of variables and constraints to be respected. Classical algorithms, such as simulated annealing, can often only find local optima and provide a non-optimal solution. Quantum optimization approaches, such as quantum annealing, adiabatic or hybrid algorithms (such as the Quantum Approximate Optimization Algorithm (QAOA)) promise to solve problems with large parameter spaces, provide higher quality solutions, and faster solution times.

Table 4 maps the use cases to problem classes in the optimization domain. Currently, there is an emphasis on three problem classes: traveling salesman for routing problems, knapsack for many supply chain optimization problems and satisfiability problems (SAT). However, it must be noted that other important problem classes exist, e.g., graph coloring and partitioning, as well as adaptations of the problem class to quantum-feasible models are under development. In [25] it was shown that many of these NP-hard optimization problems can be mapped to an Ising-spin class formulation, making them amenable to quantum annealing and adiabatic algorithms.

Table 4 Optimization Problem Classes: Main problem classes that arose in use case description

\begin{tabular}{ll}
\hline Problem Class & Use Cases \\
\hline Traveling Salesman & Vehicle Routing (VW), Robot Production Planning (BMW), Fleet Management (BASF), \\
& Transportation Cover (Munich Re) \\
Knapsack & Demand Capacity Match (Infineon), Supply Chain Optimization (Infineon), Truck \\
& Loading (SAP), Lot Sizing (SAP) \\
Satisfiability (SAT) & Software Testing (Bosch), Vehicle Feature Testing (BMW) \\
Sequencing & Matrix Production (Siemens), Shift Scheduling (BMW) \\
\hline
\end{tabular}


The traveling salesman problem aims to identify the shortest path between a set of nodes, relevant on multiple scales for inbound, intra-plant and outbound logistics. The knapsack problem is a packing problem aiming to determine the optimal collection of items minimizing the weight of all items and maximizing the value. It has many applications in supply chain management (e.g., truck loading, airplane loading [26], and lot sizing). Further, it is applicable to use cases in finance, e.g., selecting assets for an optimal portfolio. Satisfiability problems aim to identify possible solutions for a set of constraints, e.g., identifying a set of vehicles to produce given option codes and respecting constraints. Sequencing problems select an optimal sequence in which jobs should be executed considering the length of all jobs and available resources. A key objective of industry 4.0 is to increase the customizability and flexibility of production (batch size of 1 ). Matrix production refers to the usage of flexible product-agnostic production cells that can be combined as needed. However, the increased flexibility also increases demands for selecting the production sequence for a given production cell layout.

QUTAC members expect a medium business impact for most optimization problems. However, the number of optimization problems in our industries is enormous. Further, due to the industrial scale, a method that improves quality or time-to-solution by a few percentage points provides significant benefits.

\subsection{Reference use cases}

While various use cases for quantum computing have been proposed and explored $[12,19,27]$, the findings only provide limited insights for hardware and software solution providers. Thus, hardware and algorithms advances are primarily driving the ecosystem and not applications. As a result, low-level benchmarks methods, e.g., randomized-gate benchmarks [28] and metrics, such as quantum volume [29], are primarily used to evaluate the performance of a quantum system.

We propose establishing a complementary, application-centric evaluation process by using high-impact industry reference use cases for evaluation and benchmark activities [30]. A reference use case comprises a description including an assessment of the business value, an analysis of the problem class, mathematical formulations, quantum and classic reference solution, verification routines, and evaluation metrics.

These reference use cases can be used for performance evaluation of entire QC stacks, allowing the assessment of application-relevant performance parameters. Figure 2 shows how industry reference use cases bridge application requirements and quantum solutions. The reference problems provide the foundation for benchmarks of different parts of the stack, e.g., for micro-benchmarks that characterize certain gate sequencing exhibited by a use case.

Use cases must satisfy the following defining requirements to be amenable as a reference problem:

- Business impact: defined as the impact of prospective quantum-induced improvements (e.g., due to improved model quality, better solutions and shorter time-to-solutions) on processes, services or products (e.g., process efficiencies, enabled product and service innovation).

- Generality: describes the adaptability of the solution to adjacent problems in other business units, companies and industries.

- Access: ensures that problems are openly visible, sufficiently abstracted, formalized and understandable through definitions in unified terminology. 


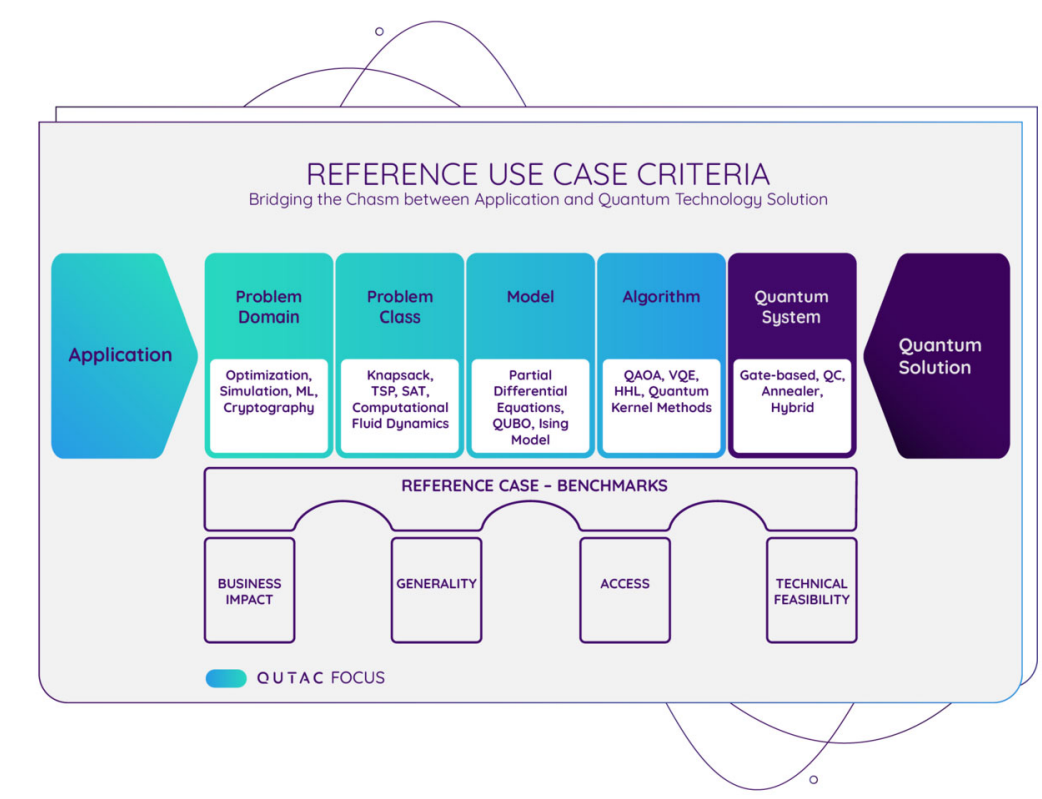

Figure 2 Reference Use Cases Criteria: Reference use cases enable holistic benchmarking involving the entire stack, i.e., quantum system, algorithm

- Technical feasibility: determines that a concise formalization and evaluation of the use case on current and future technology can be conducted and well-defined metrics are established (e.g., required computer size, solution quality, maximal solvable problem size, time-to-solution).

The QUTAC use case portfolio will serve as the basis for selecting future reference use cases. Particularly, we aim to investigate use cases that (1) have a high business impact, (2) are constrained by classical methods for optimization, simulation, and machine learning, and (3) have promising algorithmic candidates for quantum solutions.

QUTAC's target is to identify at least one reference use case per problem domain. Table 4 gives first indication for suitable reference problems, e.g., traveling salesman problems are relevant in all industries (Vehicle Routing (VW), Robot Production Planning (BMW), Fleet Management (BASF), Transportation Cover (Munich Re)).

The initial QUTAC use case portfolio will be extended to formalized use case descriptions (including data generators, reference implementation and verification routines). We will provide these as open source contribution to the community, encouraging an active engagement on these problems. We postulate that cross-industry benchmarks of reference cases will guide hardware \& software providers towards industry use cases.

\subsection{Discussion}

Quantum computing will impact many parts of the value-chains across all industries. Figure 3 illustrates areas of value creation and common problem domains amenable for quantum solutions. The most common problem domain is optimization problems with $>50 \%$ of all use cases. The majority of the optimization use cases is in the production \& logistics challenge. On average, the business impact is given as medium and the time-to-maturity as high. 


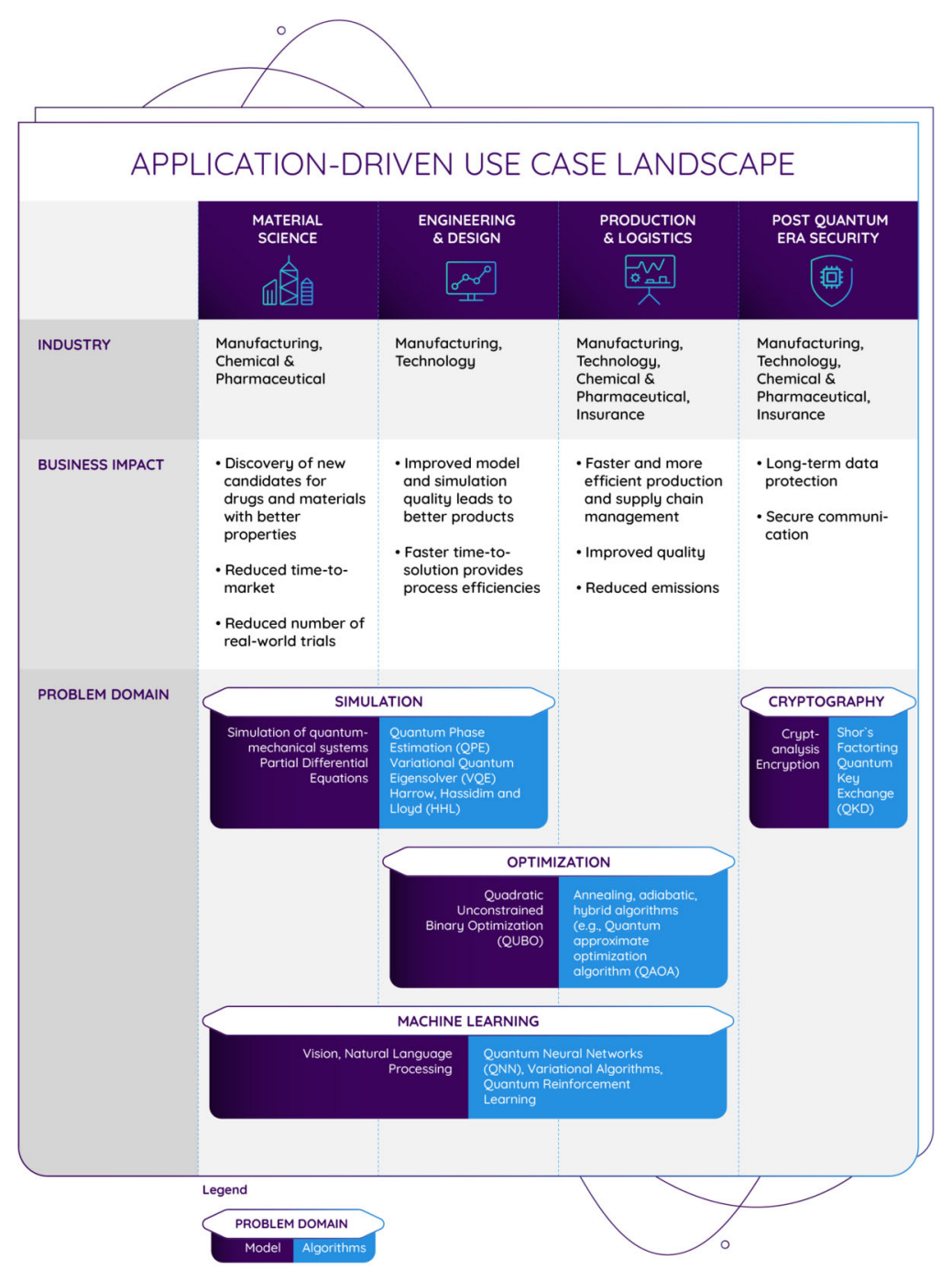

Figure 3 Application-Driven Use Case Landscape with Business Impact and Problem Domain: Quantum computing promises benefits across different value-chains, in particular material science, engineering \& design and production \& logistics. The most important problem domain is optimization with $>50 \%$ of all use cases. The majority of the optimization use cases address the production \& logistics challenge. On average, the business impact is given as medium and the time-to-maturity as high

The time-to-maturity for the simulation of quantum-mechanical systems is assessed as medium. Quantum-mechanical simulations are quantum-native problems and make up more than $20 \%$ of all use cases across multiple industry sectors, particularly, chemical and pharmaceutical industry and manufacturing. Their potential business impact is assessed as high, as they might enable the acceleration of material discovery for drug discovery and enable new products, particularly batteries. There are only two simulation use cases for engineering process support (e.g., electric drives for Bosch and computational fluid dynamics for AIRBUS). The time-to-maturity of these use cases is estimated to be high.

Artificial intelligence (AI) and machine learning are widely being adopted across industry sectors [31]. QUTAC use cases are surrogate modeling for CFD simulations obtained from fellow author AIRBUS, and reinforcement learning (Siemens). However, AI is 


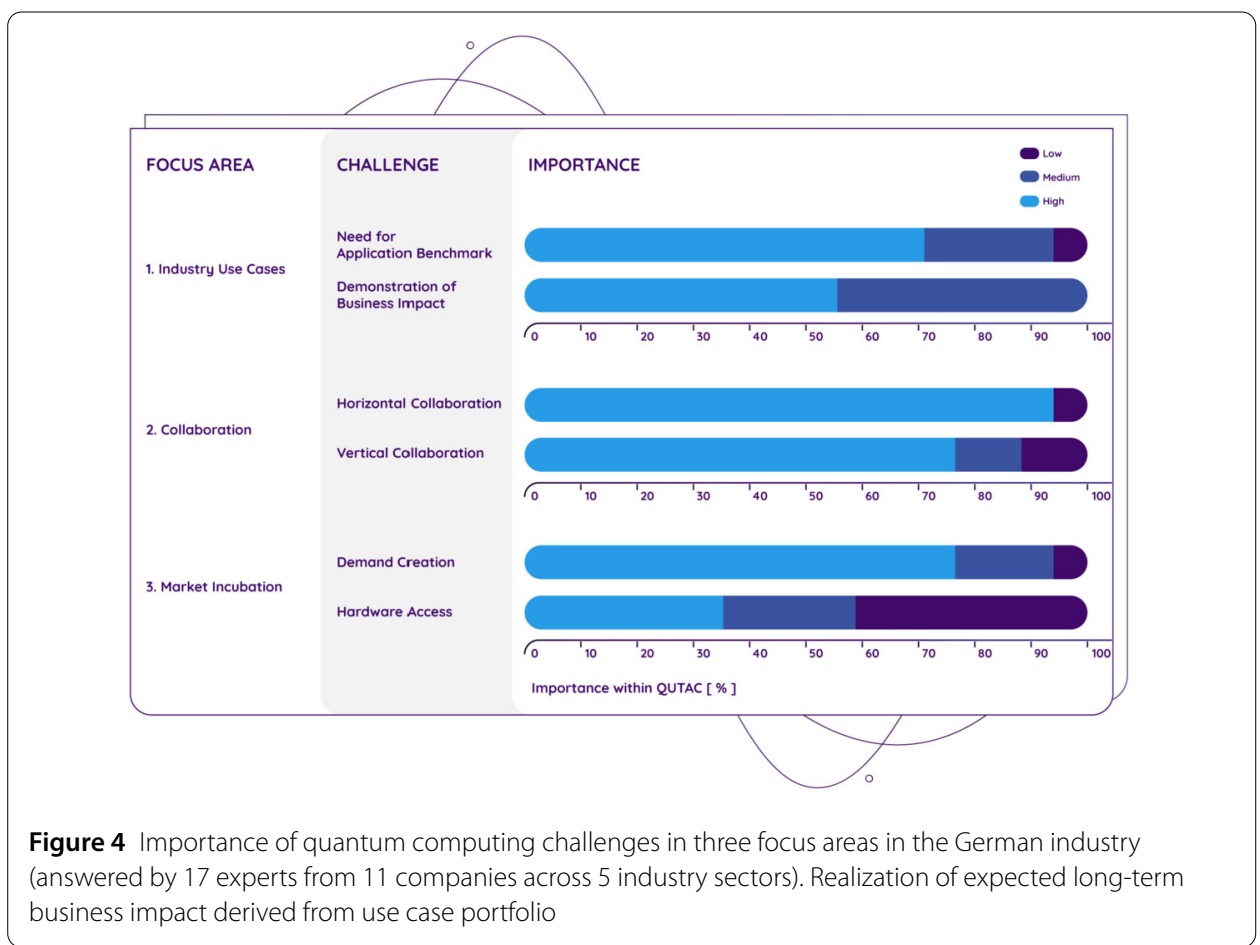

broadly applicable to almost all products and parts of the value-chain. For example, BMW lists more than $400 \mathrm{AI}$ use cases in its portfolio. That means that advances in Quantum AI will benefit many use cases [32].

\section{Challenges}

While impressive quantum supremacy results have been achieved on technical level [1,2], various challenges remain concerning transferring these results into large-scale industrial applications of quantum computing. In this section, we discuss the result of the QUTAC survey and interviews. Figure 4 illustrates the main challenges in the three focus areas: (1) industry use cases, (2) collaboration, and (3) market incubation.

\subsection{Industry use cases}

There is no proof of value for QC applications yet. The main reason is the early stage of the technology in need of fundamental research breakthroughs to allow for a scale at which business impact is tangible (see Sect. 3). The contributing application experts identified the following challenges:

- Business Impact: The contributing companies identified various use cases with medium to high impact through quantum computing. However, often a precise and proven business impact cannot be provided as this business impact critically depends on both technical (e.g., number of qubits) and business details (e.g., the value associated with certain model types). The lack of proven and coherent method for estimating business impact hinders long-term investments both by use case owner and ecosystem partners.

- Benchmark: The QC market and ecosystem are highly diverse and dynamic. Existing benchmarks primarily emphasize low-level hardware performance (e.g., gate fidelities and coherence times) and do not accurately reflect application-level performance. 
Due to a lack of community-driven application-centered benchmarks, users cannot easily infer the performance they can expect from proposed solutions. By enabling comparisons between quantum solutions, benchmarks can drive improvements on all layers of the QC stack. For example, the ImageNet [33] benchmark lead to breakthroughs in artificial intelligence and drove the creation of specialized hardware. Application benchmarks further help to establish a converged application and hardware roadmap.

- Business Integration: Transferring quantum technology solutions to business impact is complex, and in addition to a deep understanding of quantum technology, requires domain and integration expertise. For example, like data-driven use cases, QC solutions rely heavily on available data and models. Results of the quantum solution must then be translated into business outcome, e.g., by integrating them into operational systems or business decisions. In addition to quantum hardware and algorithms, business applications need holistic considerations.

\subsection{Collaboration}

To guide ecosystem activities towards industrializiation and commercialized, marketready products, an environment conducive to innovation is key. Until now, ecosystem development has been held back by traditional, rigid collaboration models in a complex stakeholder landscape, particularly between industry and research institutions. A highly intertwined technology stack and multidimensional governmental funding mechanisms create a complicated ecosystem of institutions and initiatives with many inter-dependencies and overhead. The current state makes partner contracting/sourcing a complex and lengthy process.

Funding: Agencies around the globe have been funding basic research in QC for decades (e.g., US [9, 10]). In Europe, more than 20 projects are funded as part of the Quantum Technologies Flagship [18].

Germany significantly extended its quantum program, supporting both, foundational research and industrialization of hardware and software $[16,17,34]$ with a budget of two billion Euros over five years. The quantum hubs will focus on different specific qubit technologies (e.g., ion traps or superconducting qubits). Application research is supported, e.g., via a competence network. Further, state-level initiatives in Germany have emerged, including the Munich Quantum Valley [35] and Lower Saxony Quantum Valley [36].

The resulting funding landscape is complex, fragmented and exhibits partially competing and overlapping objectives. As a result the establishment of large-scale industrialization projects will be challenging. Thus, we expect a high number of small initiatives, bearing the risk of redundancy and lack of focus. In this environment, avoiding the decoupling of application-centric industrialization and foundational research is instrumental to advance the technology at this early maturity level.

Horizontal collaboration: Survey respondents noted effective cross-industry collaboration to advance quantum computing as one of the highest-ranked challenges. Crossindustry collaboration is a crucial enabler for (1) creating a shared industry voice towards the ecosystem, (2) establishing high-impact applications that accelerate industrialization, (3) jointly facilitate activities with the emerging ecosystem, and (4) to de-risk long-term investments.

Several consortia on international level have been founded to address these challenges, e.g., QED-C [37] and QuIC [38]. Germany is missing a consortium to advocate for industry 
needs in growing German QC programs. Additionally, a framework for addressing collaboration challenges is needed, including (1) the lack of a unified terminology and standards (e.g., for use cases, benchmarks and access protocols), (2) the difficulty of sharing proprietary data and information, and (3) the lack of effective strategies to balance openness and corporate interests.

Vertical collaboration: Collaboration of industry and quantum solution providers is crucial to coherently advance applications and hardware by optimizing integration across the entire stack (hardware/software co-design). This requires the integration of industrial domain knowledge with hardware, software, and algorithmic knowledge. Setting up vertical collaborations, especially with international partners, can be challenging and requires careful consideration of IP protection, data security and privacy.

\subsection{Market incubation}

While governments, research institutions and cloud providers began to procure quantum devices, the commercial market is still in its infancy. Industrial applications with proven business impact are instrumental in establishing such markets, creating a virtuous cycle of demand and supply [39]. The contributing experts emphasizes three challenges:

Hardware Access: Access to different quantum systems is now possible through cloud service providers $[3,5]$. However, the availability, scale, and costs limits research and industrialization activities. In many cases, careful consideration of international and data protection law and vendor-specific contracts are required. Further, access is often limited, preventing low-level experiments. High-end, state-of-the-art hardware is often unavailable. As technology advances, we expect this situation to exacerbate. Another challenge is limited access to research test-beds emerging from public projects.

Demand Creation: A virtuous cycle of demand and supply is crucial to establish a new market. Additional to government-driven markets, industrial applications can serve as an important market to initiate such a cycle. In particular, application on NISQ devices useful for industry can drive demand. Market creation is a well-known challenge of deep-tech ecosystems [12], but is particularly pronounced in quantum computing due its long-term nature.

Talent \& Education: QC requires both, highly specialized and interdisciplinary skills that bridge different research fields, including engineering, industrialization, and business. Developing talent with knowledge in theoretical and applied computer science (e.g., complexity theory, operations research) and quantum computing (e.g., basics of QC operation and control, error mitigation, quantum algorithms, quantum software development kits, assessment of application-relevant hardware features) in combination with business acumen (e.g., identifying customer needs, knowledge in production and operations processes, their business and technical limitations) is seen as critical. With first applications reaching commercial viability, this situation will exacerbate.

\section{Call for action}

To advance quantum computing towards the level of industrial-scale applications, we need to act now. As we observed in other fields, e.g., artificial intelligence, early investments are essential to ensure a competitive advantage in a fast-paced digital economy. QUTAC members identified three fields of action in the focus areas: industry use cases, collaboration and market incubation, and two enablers: talent \& education and standards (Fig. 5). 


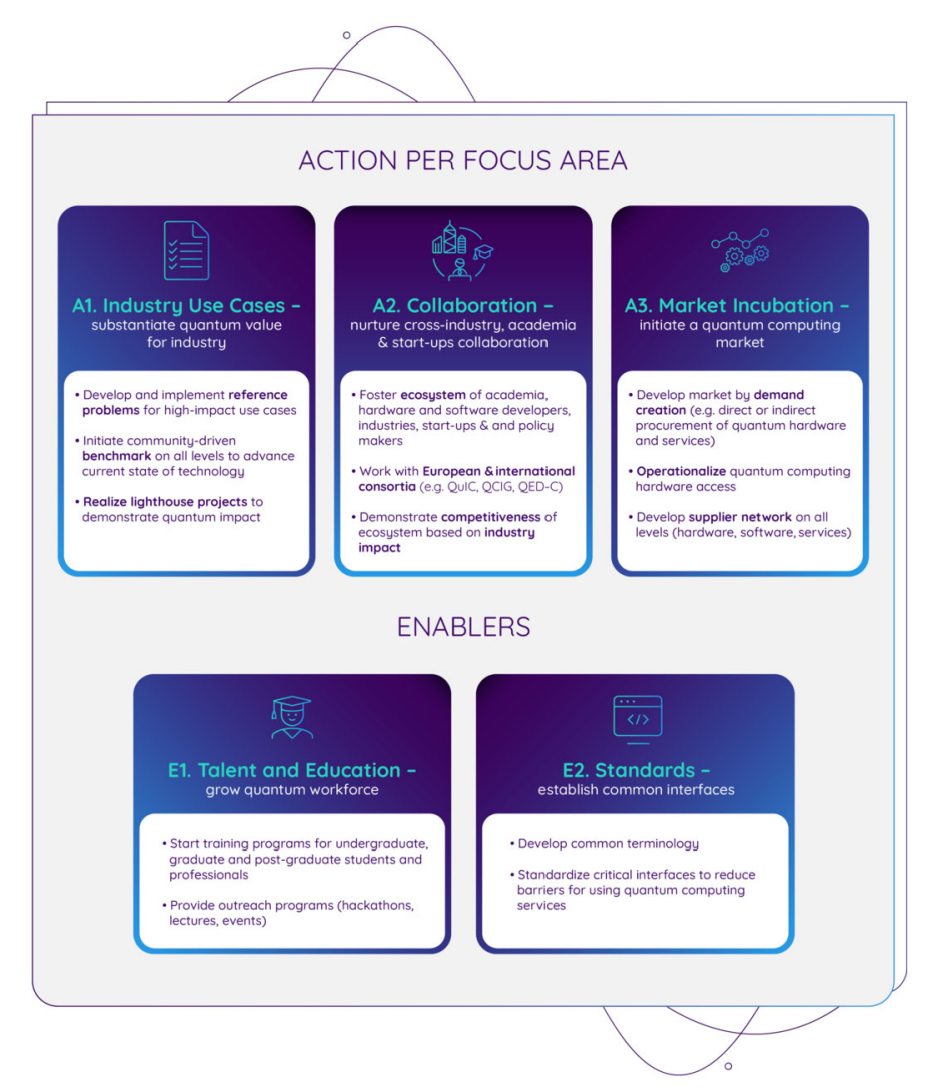

Figure 5 Action per Focus Area: We suggest three action areas (Industry Use Cases, Collaboration, and Market Incubation) to accelerate commercialization of quantum computing technology. Enablers: Talent, education and standard are crucial enablers for successful realization

A1 Industry Use Cases: To establish useful QC application on near-term devices, the industry must: (1) prioritize, develop and communicate industry reference problems and transform them to community benchmarks to steer quantum solutions towards commercial usability, (2) focus on end-to-end applications including the integration in business processes to demonstrate business impact, and (3) communicate demonstrations of quantum impact.

A2 Collaboration: European and German funding streams must be aligned to avoid redundancy. Demands and guidance for industry partners must be clearly communicated. Industry users must engage proactively in the consortia, with well-defined value propositions and contributions. Further, international collaboration and cooperation beyond Germany is vital (e.g., through QuIC [38]).

A3 Market Incubation: Commercially-useful quantum applications are vital to create new demands for quantum technologies, initiating the virtuous cycle of demand and supply. The industrialization of quantum computers to industry scale must be the long-term target, enabling profitable business models for all value-chain participants, including start-ups, components suppliers, etc.

The following enablers contribute to all action items in the three focus areas and are prerequisites for their success: 
E1 Talent $\mathcal{E}$ Education: As technology matures, the demand for quantum computing will grow. Both industry and academia must develop critical skills at the intersection of physics, engineering, computer science, and business [40], addressing undergraduate, graduate, post-graduate students, and professionals. A particular focus must be interdisciplinary skills that bridge these fields and combining low-level quantum knowledge with industry domain expertise.

E2 Standards: Entry-barriers must be lowered by development of appropriate high-level interfaces and standards (e.g., terminologies, API for accessing infrastructure). Standards are also instrumental for minimizing vendor lock-in.

The QUTAC application working group aims to advance the industrial-scale applications of QC (A1). This paper presents an initial set of cross-industry applications of quantum computing, which will provide the foundation for establishing industry reference problems. Based on these reference use cases we will establish benchmarks, which we hope will spark horizontal and vertical collaboration (A2). We actively evaluate engagement models with QuIC and other industry consortia (A2). By collaborating on community standards, e.g., a glossary, access interfaces, high-level business abstraction, we will lower the entry barriers (E2).

We work towards strengthening exchange with German and European funding agencies (A2). We specifically envision collaborative lighthouse projects that increase collaboration across the ecosystems and channel it towards high-value industrial challenges (A1, A2). By providing domain expertise, we contribute critical knowledge while benefiting from the advancements in quantum solutions. At the same time, this will generate demand for industrial quantum solutions - solving the deadlock between users and platform providers.

We postulate that QUTAC's engagement and industrial perspective will enable early markets for quantum computing technologies (A3). Partners are committed to contributing applications, data, technological and business knowledge to the emergent ecosystem (A2). Our results will be beneficial to all ecosystem participants, e.g., suppliers, system integrators, software developers, users, policymakers, funding program managers, and investors. We believe in the long-term business impact of quantum computing. We do not expect immediate value, but are convinced that it is now the time to obtain and share experience with different technologies and advance our business infrastructure to accelerate the adoption of quantum-based methods.

\author{
Acknowledgements \\ Not applicable. \\ Funding \\ Not applicable.
}

Abbreviations

Al, artificial intelligence; AQT, Alpine Quantum Technologies; BASF, Badische Anilin- und Sodafabrik (consortium partner); BCS, Boston Consulting Group; BMW, Bayerische Motoren Werke (consortium partner); FEM, finite element method; $\mathrm{HHL}$, Harrow, Hassidim and Lloyd; IBM, International Business Machines Corporation; IoT, internet of things; IQM, IQM Quantum Computers; NISQ-era, noisy intermediate-scale quantum era; NP, nondeterministic polynomial time; PVC, polyvinyl chloride; QAOA, quantum approximate optimization algorithm; QaRL, Quantum-assisted Reinforcement Learning; QC, quantum computing; QCIG, Quantum Compouting Industry Group; QED-C, Quantum Economic Development Consortium; QKD, quantum key exchange; QoMP, Quantum-optimized Matrix Production; QNN, quantum neural network; QPE, quantum phase estimation; QUBO, quadratic unconstrained binary optimization; QulC, European Quantum Industry Consortium; QUTAC, Quantum Application and Technology Consortium; SAP, Systemanalyse Programmentwicklung (consortium partner); SAT problem, satisfiability problem; VQE, variational quantum eigensolver; WW, Volkswagen (consortium partner). 


\section{Declarations}

\section{Competing interests}

Thomas Strohm is Guest Editor of EPJQT but was explicitly not involved in the review process, to avoid conflicting interests. The authors declare that they have no competing interests.

\section{Authors' contributions}

$\mathrm{AL}$ and JK proposed the main idea of the paper and prepared the manuscript. All authors have contributed use cases from their respective companies. All authors read and approved the final manuscript.

\section{Author details}

${ }^{1}$ QUTAC, Germany. ${ }^{2}$ BASF SE, Ludwigshafen, Germany. ${ }^{3}$ BMW AG, Munich, Germany. ${ }^{4}$ C.H. Boehringer Sohn AG \& Co. KG, Ingelheim, Germany. ${ }^{5}$ Robert Bosch GmbH, Stuttgart, Germany. ${ }^{6}$ Infineon Technologies AG, Neubiberg, Germany. ${ }^{7}$ Merck KGaA, Darmstadt, Germany. ${ }^{8}$ Munich Re AG, Munich, Germany. ${ }^{9}$ SAP SE, Walldorf, Germany. ${ }^{10}$ Siemens AG, Munich, Germany. ${ }^{11}$ Volkswagen AG, Wolfsburg, Germany. ${ }^{12}$ AIRBUS (external contributor to QUTAC), Ottobrunn Germany.

\section{Publisher's Note}

Springer Nature remains neutral with regard to jurisdictional claims in published maps and institutional affiliations.

\section{Received: 2 September 2021 Accepted: 22 October 2021 Published online: 13 November 2021}

\section{References}

1. Arute F et al. Quantum supremacy using a programmable superconducting processor. Nature. 2019;574:505-10

2. Zhong H-S et al. Quantum computational advantage using photons. Science. 2020;370(6523):1460-3.

3. IBM. Quantum computing. 2021. https://www.research.ibm.com/quantum-computing/.

4. D-Wave. Leap, the quantum cloud service build for business. 2021. https://www.dwavesys.com/take-leap.

5. Amazon. Amazon Braket: explore and experiment with quantum computing. 2021. https://aws.amazon.com/braket/.

6. Microsoft. Azure Quantum. 2021. https://azure.microsoft.com/services/quantum/.

7. Knight P, Walmsley I. UK national quantum technology programme. Quantum Sci Technol. 2019;4(4):040502.

8. Raymer MG, Monroe C. The US national quantum initiative. Quantum Sci Technol. 2019;4(2):020504.

9. Department of Energy. US announce over \$1 billion in awards for artificial intelligence and quantum information science research institutes. 2020. energy.gov/articles/white-house-office-technology-policy-national-science-foundation-and-department-energy

10. Malik JAN. NSF kicks off quantum leap challenge institutes program. Mater Res Soc Bull. 2020;45(3):168-9.

11. McKinsey\& Company. McKinsey quantum computing monitor. 2020. https://www.mckinsey.de/news/presse/quantum-computing-monitor-marktanalyse-investitionen.

12. Langione M, Tillemann-Dick C, Kumar A, Taneja V. Where will quantum computers create value and when. Boston Consulting. 2019. https://www.bcg.com/publications/2019/quantum-computers-create-value-when.

13. Henning Kagermann, Süssenguth F, Körner J, Liepold A. The innovation potential of second-generation quantum technologies. 2020. https://www.acatech.de/publikation/innovationspotenziale-der-quantentechnologien/.

14. Federal Ministry of Education and Research. Quantum technologies - from basic research to market (a federal government framework programme). 2018. https://www.bmbf.de/upload_filestore/pub/Quantum_technologies.pdf.

15. European Commission. Green paper on innovation. 1995. https://europa.eu/documents/comm/green_papers/pdf/com95_688_en.pdf.

16. Barz S, Leibinger P, et al. Roadmap Quantencomputing. 2021. https://www.quantentechnologien.de/fileadmin/ public/Redaktion/Dokumente/PDF/Publikationen/Roadmap-Quantencomputing-bf-C1.pdf.

17. Federal Ministry of Economic Affairs and Energy. Quantum Computing - Applications for Industry. 2021. https:// www.digitale-technologien.de/DT/Navigation/EN/Foerderaufrufe/Quanten_Computing/quanten_computing.html;

18. Commission E. Quantum technologies flagship. 2021. https://ec.europa.eu/digital-single-market/en/eu-funded-projects-quantum-technology.

19. Budde F, Volz D. The next big thing? Quantum computing's potential impact on chemicals. McKinsey \& Company 2019. https://www.mckinsey.com/industries/chemicals/our-insights/the-next-big-thing-quantum-computingspotential-impact-on-chemicals.

20. Freeman AJ. Materials by design and the exciting role of quantum computation/simulation. J Comput Appl Math 2002;149(1):27-56.

21. Capdevila-Cortada M. Electrif Haber-Bosch Nat Catal. 2019;2(3):1055.

22. Burke W. High performance computing at BMW. 2014. https:/www.slideshare.net/opendatacenter/forecast14-bp2-finalfrombmw.

23. AIRBUS. Quantum challenge: computational fluid dynamics (CFD) on quantum computers. AIRBUS. 2019. https://www.airbus.com/content/dam/corporate-topics/innovation/quantum-computing-challenge/AirbusQuantum-Computing-Challenge-PS2.pdf.

24. AIRBUS. Quantum challenge: wingbox design optimisation. AIRBUS. 2019. https://www.airbus.com/content/dam/corporate-topics/innovation/quantum-computing-challenge/AirbusQuantum-Computing-Challenge-PS4.pdf.

25. Lucas A. Ising formulations of many np problems. Front Phys. 2014;2.

26. AIRBUS. Quantum challenge: aircraft loading optimisation. AIRBUS. 2019. https://www.airbus.com/content/dam/corporate-topics/innovation/quantum-computing-challenge/AirbusQuantum-Computing-Challenge-PS5.pdf. 
27. Carrel-Billiard M, Garrison D, Dukatz C. Think beyond ones and zeros: quantum computing now. Accenture. 2017. https://www.accenture.com/_acnmedia/PDF-54/Accenture-807510-Quantum-Computing-RGB-V02.pdf.

28. Magesan E, Gambetta JM, Emerson J. Scalable and robust randomized benchmarking of quantum processes. Phys Rev Lett. 2011;106(18):180504.

29. Cross AW, Bishop LS, Sheldon S, Nation PD, Gambetta JM. Validating quantum computers using randomized model circuits. Phys Rev A. 2019:100:032328.

30. Luckow A, Klepsch J, Pichlmeier J. Towards industry reference problems. Digit Welt. 2021;2:38-45.

31. Balakrishnan T, Cui M, Hall B, Henke N. The state of ai in 2020. McKinsey \& Company. 2020. https://www.mckinsey. com/business-functions/mckinsey-analytics/our-insights/global-survey-the-state-of-ai-in-2020.

32. BMW Group. Seven principles for Al: BMW group sets out code of ethics for the use of artificial intelligence. 2020. https://www.press.bmwgroup.com/global/article/detail/T0318411EN/seven-principles-for-ai:-bmw-group-sets-outcode-of-ethics-for-the-use-of-artificial-intelligence.

33. Deng J, Dong W, Socher R, Li L-J, Li K, Fei-Fei L. ImageNet: a large-scale hierarchical image database. In: CVPR09. 2009.

34. Federal Ministry of Economic Affairs and Energy. DLR teams up with industry to develop German quantum computers. 2021. https://www.dlr.de/content/en/articles/news/2021/02/20210511_dlr-teams-up-withindustry-to-develop-german-quantum-computers.html.

35. Munich Quantum Valley (MQV). 2021. https://www.munich-quantum-valley.de.

36. Quantum Valley Lower Saxony (QVLS). 2021. https://www.qvis.de.

37. QED-C. The quantum consortium: enabling the quantum ecosystem. 2021. https://quantumconsortium.org/.

38. QulC. European Quantum Industry Consortium (QulC). 2021. https://qt.eu/about-quantum-flagship/the-quantum-flagship-community/quic/.

39. National Academies of Sciences E, Quantum Computing M. Progress and prospects. Washington: The National Academies Press; 2019.

40. Nita L, Mazzoli Smith L, Chancellor N, Cramman H. The challenge and opportunities of quantum literacy for future education and transdisciplinary problem-solving. Res Sci Technol Educ. 2021.1-17.

\section{Submit your manuscript to a SpringerOpen ${ }^{\circ}$ journal and benefit from:}

- Convenient online submission

- Rigorous peer review

- Open access: articles freely available online

- High visibility within the field

- Retaining the copyright to your article

Submit your next manuscript at $\gg$ springeropen.com 